\title{
Prevalencia de la microalbuminuria en una población hipertensa de Navarra septentrional y su relación con otros factores de riesgo cardiovascular
} Prevalence of microalbuminuria in a hypertensive population of
northern Navarre and its relation to other cardiovascular risk factors

\author{
B. Aguirre
}

\section{RESUMEN}

Objetivo. Conocer la prevalencia de microalbuminuria en una población hipertensa de Navarra Septentrional y su relación con otros factores de riesgo cardiovascular.

Pacientes y métodos. Se incluyó a todos los pacientes hipertensos conocidos de nuestro ámbito de trabajo y se diseñó un estudio transversal que valoró además la presencia de otros factores de riesgo cardiovascular, incluyendo la diabetes mellitus, la hipercolesterolemia, la obesidad, el consumo de tabaco y la ingesta de alcohol.

Resultados. Se estudiaron un total de 106 hipertensos (54,7\% mujeres). En 19 (18\%) pacientes se descubrió presencia de microalbuminuria, y un porcentaje importante presentó otros factores de riesgo cardiovascular -diabetes mellitus $(24,5 \%)$, hipercolesterolemia (61,3\%), obesidad (49\%)-. En el $12,2 \%$ concurrieron hipertensión arterial, diabetes mellitus, hipercolesterolemia y obesidad. El $84,6 \%$ de los diabéticos perteneció al tipo 2. El mayor porcentaje de factores de riesgo cardiovascular correspondió al grupo de hipertensos con microalbuminuria positiva y las diferencias más significativas se hallaron en el grupo de diabéti$\cos$.

Conclusiones. La frecuencia de microalbuminuria en nuestra población hipertensa fue similar a la obtenida en otros trabajos. Se encontró una elevada prevalencia de factores de riesgo cardiovascular, especialmente en el grupo de hipertensos con microalbuminuria positiva.

Palabras clave. Microalbuminuria. Hipertensión arterial. Riesgo cardiovascular. Diabetes mellitus.

\begin{abstract}
Aim. To determine the prevalence of microalbuminuria in a hypertense population in northern Navarre and its relationship to other cardiovascular risk factors.

Patients and methods. All the known hypertensive patients in our area of work were included, and a transversal study was designed that also evaluated the presence of other cardiovascular risk factors, including diabetes mellitus, hypercholesterolemia, obesity, smoking and alcohol drinking.
\end{abstract}

Results. A total of 106 hypertensive patients ( $54.7 \%$ women) were studied. The presence of microalbuminuria was found in 19 patients (18\%), and a significant percentage showed other cardiovascular risk factors - diabetes mellitus (24.5\%), hypercholesterolemia $(61.3 \%)$, and obesity (49\%). In $12.2 \%$ there was a concurrence of arterial hypertension, diabetes mellitus, hypercholesterolemia and obesity. Eighty-four percent of the diabetics were type 2 . The highest percentage of cardiovascular risk factors corresponded to the group of hypertensive patients with positive microalbuminuria, and the most significant differences were found in the group of diabetics.

Conclusions. The frequency of microalbuminuria in our hypertensive population was similar to that reported in other papers. A high prevalence of cardiovascular risk factors was found, especially in the group of hypertensive patients with positive microalbuminuria.

Key words. Microalbuminuria. Arterial hypertension. Cardiovascular risk. Diabetes mellitus.
Unidad de Atención Primaria en las localidades de Úrdax y Zugarramurdi. Centro de Salud de Elizondo. Navarra.

Aceptado para su publicación el 14 de julio de 2003.

\section{Correspondencia:}

Dr. Benedicto Aguirre Echeverría

Centro de Salud de Elizondo

C/ Joaquín Iriarte, 4

31700-Elizondo (Navarra)

Tfno: 948581832 - 948581833

Fax: $948581801-948599205$

e-mail: bene@terra.es 


\section{INTRODUCCIÓN}

Diversos estudios han demostrado una elevada prevalencia de hipertensión arterial (HTA), en la población general. Nuestro país, con una prevalencia del $47 \%$ ocupa el tercer lugar, después de Alemania y Finlandia en porcentaje de afectados ${ }^{1}$.

Se estima que la HTA incrementa entre dos y tres veces el riesgo de cardiopatía isquémica (angina de pecho, infarto de miocardio), de enfermedad cerebrovascular (trombosis y hemorragia cerebral), de arteriopatía periférica y de insuficiencia cardíaca ${ }^{2}$.

Por estos motivos, la HTA continúa siendo un problema clínico y de salud pública de gran magnitud. Además, es bien conocido que la HTA causa daño renal, especialmente en aquellos hipertensos que no han sido debidamente tratados y, la presencia de marcadores de lesión renal, en pacientes con factores de riesgo cardiovascular (FRV), en especial hipertensión arterial esencial, implica un peor pronósti$\mathrm{co}^{3}$.

Parving y $\mathrm{col}^{4}$ publicaron hace décadas la relación existente entre la HTA y la secreción elevada de proteínas en orina. Pero, en general, la proteinuria acompaña ya a una función renal disminuida. Por ello cobra creciente interés la más reciente descripción del valor pronóstico de la microalbuminuria (MA), que expresa una elevación anormal de la excreción urinaria de albúmina en ausencia de proteinuria clínica. La mayoría de grupos ${ }^{5-7}$ han confirmado la correlación existente entre la HTA y la existencia de MA, así como su normalización después de controlar las cifras tensionales.

Además, es preciso tener presente que un porcentaje no despreciable de personas hipertensas, son también diabéticas -la mayoría diabetes mellitus de tipo 2 (DM2)- y que las complicaciones macrovasculares y microvasculares a largo plazo, de la diabetes tipo 2 como fuente de morbimortalidad, son muy importantes.

En el estudio United Kingdom Prospective Diabetes Study (UKPDS) ${ }^{8}$ se indica que, en pacientes con hipertensión y DM2, la mejoría en el control de la presión arterial, se asoció a reducciones clínicamente significativas, de los riesgos de mortalidad relacionados con diabetes, de las complicaciones asociadas a diabetes, del avance de la retinopatía diabética y el deterioro de la agudeza visual.

Además, es de sobra conocido el rápido aumento de la población anciana, sobre todo en los países industrializados y como dato curioso, cerca de la mitad de todas las personas con DM2 y que corresponde a la mayoría de diabéticos, son mayores de 65 años de edad ${ }^{9}$. Por consiguiente, es lógico suponer que a medida que aumente la población anciana, aumentará el número de pacientes con diabetes.

En definitiva, en los pacientes hipertensos la MA, además de ser un marcador precoz de afección renal, se correlaciona con el daño orgánico global y con el desarrollo futuro de complicaciones cardiovasculares $^{10}$.

El objetivo de este trabajo es conocer la prevalencia de microalbuminuria en la población hipertensa de nuestra área geográfica y su relación con otros factores de riesgo cardiovascular.

\section{PACIENTES Y MÉTODOS}

Se trata de un estudio de prevalencia, de todos los pacientes registrados como hipertensos en nuestra área de trabajo $(n=119)$, pertenecientes a una zona de la Navarra Septentrional que comprende las localidades de Úrdax y Zugarramurdi. La población de hecho es de 633 habitantes y el estudio valoró las cifras de tensión arterial en la población mayor de 30 años.

La mayoría de los hipertensos eran conocedores de su enfermedad y se hallaban en tratamiento farmacológico, desde un período de tiempo que oscilaba entre 6 meses y 10 años. El resto de individuos que completaron el trabajo, conocieron la enfermedad como consecuencia de los registros efectuados en el consultorio médico, unas veces al azar y en ocasiones por la existencia de algún síntoma que hacía sospechar su presencia.

El tiempo transcurrido en la agrupación de todos los pacientes que formaron parte de la muestra, fue de dos años apro- 
ximadamente y en todos los casos se cumplió el criterio de inclusión, en concordancia a los acuerdos que se expresan en la $1^{a}$ Guía Europea para el manejo de la Hipertensión Arterial. Se incluyó en el estudio a todas las personas que después de tres registros, separados como mínimo en intervalos de tiempo de una semana, presentaron valores de presión arterial sistólica superior a $140 \mathrm{mmHg}$ y/o una presión diastólica superior a $90 \mathrm{mmHg}$. La determinación de las cifras de presión arterial se realizó en el consultorio médico, con los pacientes sentados, en condiciones basales y mediante el empleo de un esfigmomanómetro de mercurio, situado a la altura del corazón.

Se practicó una determinación de MA en orina matinal, para lo cual la enfermera de la localidad se ocupó de adiestrar a los pacientes, en la sistemática de la técnica de recogida, y con este fin entregó un frasco estéril a cada uno de los hipertensos, descartándose previamente las causas de proteinuria extrarrenal (insuficiencia cardiaca congestiva, enfermedad febril aguda, hematuria, infección urinaria, ejercicio vigoroso y la existencia de un desequilibrio ácido-base). Para su evaluación, se empleó el modelo de analizador Hitachi917 y se utilizó el reactivo Tina-quant-aAlbúmina, considerándose MA positiva a los valores de albuminuria comprendidos entre $30-300 \mathrm{mg} / \mathrm{l}$.

Los valores de creatinina, obtenidos en análisis de sangre matinal y en ayunas, se estimaron patológicos cuando las cifras eran superiores a $1,2 \mathrm{mg} / \mathrm{dl}$.

De igual modo, mediante el análisis de sangre matinal practicado en ayunas, se obtuvieron los valores de colesterol total y se consideró hipercolesterolemia cuando las cifras eran iguales o superiores a 220 $\mathrm{mg} / \mathrm{dl}$.

En todos los hipertensos incluidos se consideró la existencia de diabetes mellitus (DM). El registro de la glucemia se efectuó en la sangre periférica de los pacientes, extraída en ayunas y se diagnosticó a todos los individuos cuyas cifras de glucemia, obtenidas en dos o más análisis repetidos, de intervalos semanales, eran igual o superiores a $126 \mathrm{mg} / \mathrm{dl}$, y también cuando en una determinación aislada o tras sobrecarga oral de glucosa se obtuvo un valor igual o superior a $200 \mathrm{mg} / \mathrm{dl}$. Se contemplaron dos grupos de individuos: los diabéticos tipo 1 o dependientes de insulina y los de tipo 2, que respondían al uso de antidiabéticos orales y por tanto no estaban vinculados al tratamiento con insulina.

Se consideró que los hipertensos presentaban elevado consumo de cigarrillos, cuando el número de pitillos fumados era igual o superior a 20 diarios, en un espacio de tiempo de al menos 6 meses antes de su inclusión en el estudio.

El consumo de alcohol se consideró elevado cuando la ingesta era superior a $40 \mathrm{~g} /$ día en los varones y excedía de 24 $\mathrm{g} /$ día en las mujeres, al menos hasta 6 meses antes de su inclusión en nuestro trabajo.

Finalmente, para la estimación de la obesidad se tuvo presente el índice de masa corporal (IMC) según la fórmula: IMC $=$ Peso $(\mathrm{Kg}) /[\text { Talla }(\mathrm{m})]^{2}$. Se calificó de obeso a todo individuo cuyo IMC era igual o superior a 30 .

En el estudio estadístico, las variables cuantitativas se presentan como media, con desviación típica y el rango, mientras que las variables cualitativas se indican como porcentaje. Se ha utilizado la "t" de student para las variables cuantitativas y la $\mathrm{c}^{2}$ para las variables cualitativas.

\section{RESULTADOS}

Se comenzó el trabajo estudiando a 119 pacientes con HTA y finalmente concluyeron el estudio 106 individuos, que representan al 16,7\% de la población de nuestra área geográfica $-48(45,3 \%)$ eran varones y $58(54,7 \%)$ mujeres-. La media de edad fue de 62,4 años (desviación típica=11,8; intervalo=35-88 años). La edad media de los hombres era de 59,2 años y el de las mujeres de 65,1 años, con diferencias significativas entre los dos sexos $(p=0,01)$. De todos ellos, 90 (85\%) individuos tenían una edad igual o superior a 50 años y 49 (46,3\%) superaban los 65 . En el grupo de pacientes que superaba los 65 años de edad, 35 (71,5\%) correspondían al sexo femenino y $14(28,5 \%)$ eran varones. Se 
excluyó a 13 individuos, de los cuales 10 fallecieron antes de finalizar el estudio y 3 se desplazaron a otros lugares de residencia.

Se valoró la existencia de otros FRV en el total de la población hipertensa y se encontró que $26(24,5 \%)$ eran diabéticos, de los cuales $22(20,7 \%)$ correspondían a DM2 y sólo $4(3,7 \%)$ eran DM1. Un elevado porcentaje tenía cifras elevadas de colesterol y/o eran obesos y sin embargo, muy pocos consumían alcohol o presentaban un elevado consumo de tabaco (Tabla 1). Finalmente, en 13 individuos que representaban al 12,2\% del total de hipertensos, concurría la presencia de HTA, DM, hipercolesterolemia y obesidad.

A excepción del escaso número de pacientes hipertensos que además eran severos consumidores de tabaco y/o bebe- dores, encontramos que el mayor porcentaje de individuos con presencia de otros FRV, correspondió al grupo de pacientes con MA positiva, si lo comparamos con el grupo de hipertensos y MA negativa. Destacó, por su interés epidemiológico, la elevada concurrencia existente entre hipertensos con MA positiva y DM, confirmándose además diferencias muy significativas en los dos grupos de hipertensos estudiados (Tabla 2).

La edad media de los pacientes con MA positiva era de 66,5 años y la de los individuos con MA negativa era de 61,4 años. No existieron diferencias significativas entre ambos grupos de pacientes ( $t$ de Student $=1,72 ; \mathrm{p}=0,9)$.

En el conjunto de hipertensos con MA negativa, $72(82,7 \%)$ pacientes tenían una edad igual o superior a los 50 años mien-

Tabla 1. Características generales de la población hipertensa estudiada.

\begin{tabular}{lcc}
\hline & NÚMERO & FRECUENCIA (\%) \\
\hline Sexo: & & 45,3 \\
$\quad$ - Hombres & 48 & 54,7 \\
$\quad$ - Mujeres & 58 & 24,5 \\
Diabetes mellitus (glucemia $>126 \mathrm{mg} / \mathrm{dl}$ ) & 26 & 61,3 \\
Hipercolesterolemia $\geq 220 \mathrm{mg} / \mathrm{dl}$ & 65 & 49 \\
Obesidad $(\mathrm{IMC}>30)_{\text {Fumadores }}^{1}$ & 52 & 2,8 \\
Bebedores $^{2}$ & 3 & 3,8 \\
Creatinina $>1,2 \mathrm{mg} / \mathrm{dl}_{\text {Microalbuminuria positiva }}{ }^{3}$ & 4 & 16 \\
Proteinuria positiva $^{4}$ & 17 & 17,9 \\
\hline
\end{tabular}

IMC: índice de masa corporal; Fumadores ${ }^{1}$ : consumo de tabaco $>20$ cigarrillos/24h; Bebedores ${ }^{2}$ : consumo de alcohol $>40 \mathrm{~g} /$ día en varones y $>24 \mathrm{~g} /$ día en mujeres; Microalbuminuria ${ }^{3}$ positiva: albuminuria=30-300 mg/l; Proteinuria ${ }^{4}$ positiva: albuminuria $>300 \mathrm{mg} / \mathrm{l}$.

Tabla 2. Características generales de los grupos de hipertensos con microalbuminuria positiva y negativa.

\begin{tabular}{lcccc}
\hline & MA POSITIVA (N=19) & MA NEGATIVA (N=87) & $\chi \mathbf{2}$ & P \\
\hline Sexo: & $8(42 \%)$ & $40(46 \%)$ & 0,1 & 0,7 (NS) \\
$\quad$ - Hombres & $11(58 \%)$ & $47(54 \%)$ & & \\
$\quad$ - Mujeres & $11(58 \%)$ & $15(17 \%)$ & 13,7 & 0,0001 \\
Diabetes mellitus (glucemia $>126 \mathrm{mg} / \mathrm{dl})$ & $14(74 \%)$ & $51(58,6 \%)$ & 1,5 & 0,2 (NS) \\
Hipercolesterolemia $\geq 220 \mathrm{mg} / \mathrm{dl}$ & $13(68 \%)$ & $39(45 \%)$ & 3,3 & 0,07 (NS) \\
Obesidad $(\mathrm{IMC}>30)_{\text {Fumadores }^{1}}$ & $0(0 \%)$ & $3(3,5 \%)$ & $*$ & 1 (NS) \\
Bebedores $^{2}$ & $1(5 \%)$ & $3(3,5 \%)$ & $*$ & 0,5 (NS) \\
Creatinina $>1,2 \mathrm{mg} / \mathrm{dl}$ & $4(21 \%)$ & $13(15 \%)$ & 0,4 & 0,5 (NS) \\
\hline
\end{tabular}

MA: microalbuminura; IMC: índice de masa corporal; Fumadores ${ }^{1}$ : consumo de tabaco $>20$ cigarrillos/24h; Bebedores ${ }^{2}$ : consumo diario de alcohol $>40 \mathrm{~g} /$ día en varones y $>24 \mathrm{gr} /$ día en mujeres *: se emplea el test estadístico de Fisher; NS: no significativo. 
Tabla 3. Presencia de dos o más factores de riesgo vascular, en los hipertensos con microalbuminuria positiva y negativa.

\begin{tabular}{lcccc}
\hline & MA POSITIVA (N=19) & MA NEGATIVA (N=87) & $\boldsymbol{\chi 2}$ & P \\
\hline HTA+Colesterol+Obesidad & $10(52,7 \%)$ & $22(25,3 \%)$ & 5,5 & 0,02 \\
\hline HTA+Diabetes mellitus+Colesterol & $7(37 \%)$ & $12(13,8 \%)$ & 4,2 & 0,04 \\
\hline HTA+Diabetes mellitus +Obesidad & $7(37 \%)$ & $10(11,5 \%)$ & 5,7 & 0,02 \\
\hline HTA+ Diabetes mellitus +Colesterol+Obesidad & $5(26,4 \%)$ & $8(9,2 \%)$ & 2,8 & 0,1 (NS) \\
\hline
\end{tabular}

MA: microalbuminuria =30-300 mg/l; HTA: hipertensión arterial; colesterol total > $220 \mathrm{mg} / \mathrm{dl}$; Obesidad: índice de masa corporal >30; diabetes mellitus $>126 \mathrm{mg} / \mathrm{dl}$; NS: no significativo.

tras que $15(17,2 \%)$ tenían una edad inferior. En este grupo, hallamos elevación de las cifras de creatinina en 13 (15\%) pacientes, todos de edad igual o superior a los 50 años, de los cuales sólo 3 eran diabéticos.

También observamos que la incidencia de FRV de la población hipertensa, era igualmente elevada cuando se contempló la concurrencia de dos o más factores en un mismo individuo, especialmente en los pacientes que tenían MA positiva, en comparación con el grupo de hipertensos y MA negativa (Tabla 3).

En el grupo de pacientes con MA positiva, menos en el caso de una mujer de 38 años que correspondía a la primera de las coaliciones (HTA+colesterol+obesidad), en el resto de alianzas de FRV, todos los pacientes tenían una edad igual o superior a los 50 años.

De igual forma, en el grupo de hipertensos con MA negativa que tenían dos o más FRV, excepto la primera agrupación (HTA+colesterol+obesidad) en la que existían 4 individuos con menos de 50 años, en el resto de coaliciones de FRV todos tenían una edad igual o superior a los 50 años.

\section{DISCUSIÓN}

En 1974, Parving y col ${ }^{4}$ comunicaron la relación existente entre los pacientes hipertensos insuficientemente tratados y la presencia de albúmina en orina. Además confirmaron la relación existente entre los niveles de albúmina en orina y los valores de TA, de modo que la albuminuria descendía cuando se lograban controlar las cifras tensionales.

La prevalencia de MA en la población hipertensa es muy variable según los diferentes trabajos publicados. Estudios epi- demiológicos establecen una prevalencia de MA en los hipertensos que oscila entre el 6 y el $24 \%{ }^{11}$. Según otros ${ }^{12}$, este porcentaje puede superar el $40 \%$ de la población hipertensa. En nuestro grupo de hipertensos, hallamos 19 (18\%) pacientes con MA positiva -8 varones y 11 mujeres-, porcentaje similar al obtenido en trabajos más amplios $^{13-19}$ (Tabla 4); si bien es preciso tener en cuenta que en nuestra casuística, todos los hipertensos se hallaban en tratamiento farmacológico con anterioridad a su inclusión en el estudio y además la MA obtenida fue el resultado de un único análisis de orina. Sin embargo, en todos los casos en que la MA resultó positiva, las cifras de TA de estos pacientes eran por lo general elevadas, con valores que oscilaban entre $150-170 \mathrm{~mm} / \mathrm{Hg}$ de presión sistólica y $87-100 \mathrm{~mm} / \mathrm{Hg}$ de diastólica y además, como se demuestra, un porcentaje elevado presentaba otros FRV asociados.

Tabla 4. Prevalencia de microalbuminuria en hipertensión arterial (Bonet et $\mathrm{al}^{13}$ ).

\begin{tabular}{lc}
\hline AUTOR & PREVALENCIA \\
\hline Bigazzi y col & \\
\hline Coltone y col $^{15}$ & $40 \%$ \\
\hline Hoegholm y col $^{16}$ & $37 \%$ \\
\hline Redon y col & $13 \%$ \\
\hline Pontremoli y col $^{18}$ & $22 \%$ \\
\hline Spangler y col & \\
\hline
\end{tabular}

En los últimos años se está considerando la MA como un factor de riesgo cardiovascular, de modo que los pacientes en los que se detecta, presentan una supervivencia inferior a los que no la tienen ${ }^{7}$, si bien no es sencillo afirmar en estadios iniciales si la MA es un factor predictivo por sí 
mismo o lo verdaderamente importante es su asociación con otros FRV. Sin embargo parece que no existe duda de que cualquier otro FRV asociado a la HTA, incrementa las posibilidades de daño vascular.

Se ha descrito la existencia de diversos factores que favorecen la producción de MA en los pacientes con $\mathrm{HTA}^{20}$. Algunos autores han señalado la asociación existente entre la MA, el hábito tabáquico y la ingesta de alcohol ${ }^{5,21}$. Curiosamente, nosotros no hallamos esta concurrencia y sólo un paciente (5\%) varón que presentó MA positiva era consumidor elevado de alcohol, y en ninguno se halló hábito tabáquico severo.

Sin embargo, en nuestro trabajo encontramos una notable asociación entre la presencia de albúmina en orina y la existencia de otros FRV asociados a la hipertensión arterial, existiendo una fuerte concurrencia entre la MA, la DM, la hipercolesterolemia, la obesidad y la edad de los pacientes incluidos en el estudio. Así, en el grupo de pacientes con MA positiva, menos en el caso de una mujer de 38 años, el resto (95\%) superó los 50 años de edad; más de la mitad (58\%) eran diabéticos -la mayoría (47,4\%) DM2-, en 14 (74\%) pacientes se encontró hipercolesterolemia y 13 (68\%) eran obesos.

Algunas series ${ }^{6,22}$ asocian la MA con un incremento en la prevalencia de FRV. Esta circunstancia tiene mucho interés porque, como es de sobra conocido, la HTA y la DM están implicadas en el daño del endotelio vascular y en numerosas ocasiones estas patologías coinciden en un mismo individuo, como se muestra en nuestro estudio. La excreción urinaria de albúmina en los pacientes hipertensos, representa un estadio precoz de deterioro renal propio de la enfermedad y se relaciona con una mayor morbi-mortalidad por enfermedad cardiovascular. Esta relación cobra aún mayor interés si, como sucede en nuestra serie, existe un elevado número de pacientes en los que concurren dos o más FRV.

A diferencia de lo que ocurre en la DM1, en los pacientes con DM2 no existe una relación clara entre la MA y las lesiones microvasculares. Sin embargo, la presencia de MA se ha relacionado como un importante factor predictivo de enfermedad macrovascular ${ }^{23,24}$. Se han barajado algunas causas como responsables de esta relación y se ha observado, al igual que sucede en nuestro trabajo, que los pacientes con microalbuminuria tienen unas cifras de presión arterial ${ }^{25} \mathrm{y}$ unos niveles de lípidos ${ }^{26,27}$ más elevados.

Cuando en un mismo individuo concurren HTA y DM, algunos autores ${ }^{28}$ insisten en la necesidad de lograr descensos de los valores de TA, por debajo de los niveles hasta ahora consensuados, hasta cifras de $130 / 80$, e incluso hasta cifras inferiores cuando se evidencia una proteinuria superior a $1 \mathrm{~g} / 24 \mathrm{~h}$. Diversos trabajos ${ }^{29.31}$ realizados en pacientes diabéticos, han demostrado una disminución de los eventos cardiovasculares en los individuos que recibían un tratamiento más intensivo, con el objetivo de descender las cifras de TA hasta valores de $80 \mathrm{mmHg}$ de TA diastólica. En nuestro grupo de pacientes hipertensos, en ningún caso se tuvo presente un descenso de las cifras de TA por debajo de 130/80 en el mejor de los casos.

Nuestros hallazgos, en concordancia con importantes estudios epidemiológicos, revelaron una elevada prevalencia de hipercolesterolemia en la población hipertensa. El porcentaje que se describe en algunos trabajos ${ }^{32,33}$ oscila desde el $35 \%$ en hipertensos mayores de 40 años con hipercolesterolemias superiores a $250 \mathrm{mg} / \mathrm{dl}$, hasta el $81 \%$ de la población hipertensa, cuando se considera el valor del colesterol por encima de $200 \mathrm{mg} / \mathrm{dl}$. En nuestro país, cerca del $40 \%$ de los hipertensos presenta cifras de colesterol superiores a 240 $\mathrm{mg} / \mathrm{dl}^{34,35}$. Nosotros hallamos hipercolesterolemia igual o superior a $220 \mathrm{mg} / \mathrm{dl}$ en el $61,33 \%$ de la población hipertensa. Además, se constató un porcentaje elevado de colesterol total (74\%) en pacientes con HTA y MA positiva.

No hemos hallado trabajos que justifiquen con certeza la existencia de niveles elevados de colesterol, en los pacientes con HTA y MA positiva. Nuestros hallazgos podrían hallar acreditación en la idiosincrasia y la tradición gastronómica de la población estudiada; pero también se ha descrito la posibilidad de que la MA favo- 
rezca la dislipemia y en este sentido se sabe que las pérdidas proteicas, favorecen el aumento de los niveles de colesterol y de lipoproteína-a ${ }^{36,37}$ y por otro lado se conoce que las dislipemias ocasionan $\mathrm{MA}^{38}$. En cualquier caso, es sabido que el daño vascular en presencia de MA se incrementa, cuando se asocia la existencia de hipercolesterolemia. Del mismo modo, el tratamiento de la dislipemia tiende, no sólo a prevenir el daño vascular, sino también a reducir la microalbuminuria ${ }^{39,40}$.

Existen trabajos ${ }^{41,42}$ que relacionan la existencia de MA con la edad avanzada y el predominio del sexo masculino. En nuestro trabajo no hallamos diferencias significativas entre ambos sexos, sin embargo cuando estudiamos la edad de los pacientes comprobamos que, excepto en el caso de una mujer de 38 años, el resto tenía una edad superior a los 50. Esta circunstancia tiene especial interés por cuanto en los pacientes hipertensos y añosos, es más elevada la prevalencia de la HTA sistólica, al tiempo que se incrementa la rigidez de los vasos, circunstancias ambas que contribuyen al deterioro de la pared vascu$\operatorname{lar}^{43,44}$. En nuestro estudio, el 58\% de los pacientes con HTA y MA positiva tenían una edad igual o superior a los 65 años.

De todos los factores de riesgo, la edad y el sexo son los únicos que no pueden ser modificados y también con la edad aumenta la probabilidad de dislipemia, sobrepeso, HTA, DM y disfunción renal. Además como consecuencia de los cambios fisiopatológicos que existen en la edad avanzada, se produce hiperfiltración renal y es más probable la aparición de MA, especialmente si se añaden otros FRV, como es frecuente y también sugieren nuestros resultados.

Es sabido que un elevado porcentaje de la población hipertensa es obesa, como demuestran nuestros hallazgos, donde encontramos esta asociación en el $49 \%$ de los hipertensos estudiados. En la literatura médica, son numerosos los trabajos ${ }^{45-48}$ en los que se relaciona el peso corporal, con la elevación de las cifras de TA en la edad adulta y también se indica que la obesidad es el más fuerte predictor de DM y de otros FRV para un mismo grupo de edad. Ade- más, en concordancia con otros autores ${ }^{49}$, que hallaron una fuerte concurrencia entre obesidad y la presencia de MA, en nuestro trabajo encontramos que el $68,5 \%$ de hipertensos con MA positiva eran obesos. De esta forma existe un nexo de unión que justifica en ocasiones la concurrencia de diversos FRV en un mismo individuo. Y como el sobrepeso y la obesidad se relacionan con la resistencia a la insulina, cada vez queda más patente la existencia de nexos fisiopatológicos comunes entre la hipertensión, la obesidad y otros FRV.

En diversos trabajos ${ }^{50,51}$ ha quedado demostrado que el tratamiento antihipertensivo durante períodos variables de tiempo, es capaz de reducir significativamente la MA en el enfermo con hipertensión arterial esencial, con independencia del fármaco elegido y por tanto parece indiscutible que, el principal objetivo para evitar la aparición de daño vascular en los enfermos que presentan HTA y/o DM, consiste en el control exhaustivo de las cifras tensionales y de los valores glucémicos. De igual forma se manifiestan las Sociedades Europeas de Hipertensión y Cardiología, con motivo de hacer pública la "1" Guía Europea de manejo de la Hipertensión Arterial" ${ }^{2}$. Entre las novedades que se citan, se hace especial mención al descenso de las cifras tensionales, por debajo de los valores considerados normales cuando no se asocian otros FRV y se insta además al tratamiento concomitante de cualquier factor de riesgo añadido.

Por tanto, se puede concluir diciendo que, en vista del envejecimiento de la población, el perfil del paciente hipertenso en el futuro es el de una persona mayor de 60 años, con predominio de la hipertensión arterial sistólica y en muchos casos con presencia de otros FRV como la DM, la hipercolesterolemia y la obesidad; y que por tanto, ningún esfuerzo será vano en la consecución de reducir o eliminar cualquiera de los FRV modificables, que se han considerado en este estudio.

\section{AGRADECIMIENTOS}

Quiero expresar mi gratitud a M $\mathrm{M}^{\mathrm{a}}$ Ángeles Nuin Villanueva, de la Unidad de Programación de Atención Primaria, por su 
valiosa contribución en la calidad metodológica de este trabajo y a Bernadett Mihura, enfermera diplomada, por su cooperación, las veces que he requerido su auxilio.

\section{BIBLIOGRAFIA}

1. Wolf-Maier K, Cooper RS, Banegas JR, Giampaoli S, Hense H-W, Joffres M et al. Hypertension prevalence and blood pressure levels in 6 European Countries, Canada, and the United States. JAMA 2003; 289: 2363-2369.

2. KANNEL WB. Blood presure as o cardiovascular risk factor. JAMA 1996; 275: 1571-1576.

3. PERERA GA. Hypertensive vascular disease: description and natural history. J Chronic Dis 1995; 1: 33-42.

4. Parving HH, Jensen HAE, Mogensen CE, Evrin $\mathrm{PE}$. Increased urinary albumin excretion rate in benign essential hypertension. Lancet 1974; I: 1190-1192.

5. Mogensen CE. Microalbuminuria and hypertension with focus on type 1 and type 2 diabetes. J Intern Med 2003; 254: 45-66.

6. BiAnChI S, BigazZI R, CAMPESE VM Microalbuminuria in esential hypertension: significance, pathophysiology, and therapeutic implications. Am J Kidney Dis 1999; 34: 973975.

7. Palatini P. Microalbuminuria in hypertension. Current hypertension reports 2003; 5 : 208-214.

8. UK Prospective Diabetes Study Group. Tight blood pressure control and risk of macrovascular and microvascular complications in type 2 diabetes: UKPDS 38. Br Med J 1998; 317: 703-713.

9. Gossain VV, Carella MJ, Rovner DR. Managemente of diabetes in the elderly: a clinical perspective. J Assoc Acad Minority Phys 1994; 5: 22-31.

10. HeLDELBERG W. Pathogenesis, prevention, and treatment of diabetic nephropaty. Lancet 1998; 352: 213-219.

11. Hornych A, Marre M, Mimran A, Chaignon A, ASMAR R, FAuvel JP. Microalbuminuria in arterial hypertension. Measurement, variables, interpretation, recomendations. Arch Mal Coeur Vaiss 2000; 93: 1304-1308.

12. Rosa TT, Palatini P. Clinical value of microalbuminuria in hypertension. J Hypertens 2000; 18: 645-654.

13. Bonet J, Vila J, Alsina MJ, Ancochea L, Romero R. Prevalencia de microalbuminuria en la población general de un área mediterránea española y su asociación con otros factores de riesgo cardiovascular. Med Clin (Barc) 2001; 16: 573-574.

14. Bigazzi R, Bianchi S, CAMPese VM, Baldari G. Prevalence of microalbuminuria in a large population of patients with mild to moderate essential hypertension. Nephron 1992; 62: 120.

15. Cottone S, Contorno A, Nardi E, Zagarrigo C, Mangano MT, Panepinto N et al. Prevalence of microalbuminuria in recent-onset essential hypertension] Cardiologia 1993; 38: 733-736.

16. Hoegholm A, Bang LE, Kristensen KS, Nielsen JW, HoLM J. Microalbuminuria in 411 untreated individuals with established hypertension, white coat hypertension, and normotension. Hypertension. 1994; 24: 101-105.

17. Redon J, Liao Y, Lozano JV, Miralles A, Baldo E, COOPER RS. Factors related to the presence of microalbuminuria in essential hypertension. Am J Hypertens 1994; 7: 801-807.

18. Pontremoli R, Cheli V, Sofia A, Tirotta A, RavERA M, Nicolella C et al. Prevalence of microand macroalbuminuria and their relationship with other cardiovascular risk factors in essential hypertension. Nephrol Dial Transplant 1995; 10: 6-9

19. Spangler JG, Bell RA, Summerson JH, Konen JC. Correlates of abnormal urinary albumin excretion rates among primary care patients with essential hypertension. J Am Board Fam Pract 1997; 10: 180-184.

20. Mimran A, RibTeinJ, DuCailer G. Microalbuminuria in essential hypertension. Cup Opin Nephrol Hypertens 1999; 8: 359-363.

21. Ticket J, Vol S, Hallab M, Caces F, Marre M. Epidemiology of microalbuminuria in a french population. J Diab Compl 1994; 8: 294295.

22. Gerstein HC, Mann JFE, Yi Q, Zinman B, DinNEEN SF, HoOgwerf B et al. Albuminuria and risk of cardiovascular events, death, and heart failure in diabetic and nondiabetic individuals. JAMA 2001; 286: 421-426.

23. Matock MB, Morrish NJ, Viberti GC, Keen H, FITGERALD AP. Prospective study of microalbuminuria as predictor of mortality in NIDDM. Diabetes 1992; 41: 736-741.

24. Zamora A, Marrugat J. Pronóstico de los pacientes diabéticos con cardiopatía isquémica. Rev Esp Cardiol 2002; 55: 751-762.

25. Schmitz A, VAeth M, Mogensen CE. Systolic blood pressure relates to the rate of progression of albuminuria in NIDDM. Diabetologia 1994; 37: 1251-1258.

26. Campese V, Bianchi S, Bigazzi R. Association between hyperlipidemia and microalbumin- 
uria in essential hypertension. Kidney Int 1999; 71: S10-13.

27. Halimi JM, Forhan A, Balkau B, NovaK M, WiLPART E, TicheT J et al. Is microalbuminuria an integrated risk marker for cardiovascular disease and insulin resistance in both men and women? J Cardiovasc Risk 2001; 8: 139146.

28. GonzÁlez JR, Alegría E, García JM, GonzÁlez I, VICENTE J. Impacto de la diabetes en las enfermedades cardíacas en España. Estudio CARDIOTENS. Med Clin (Barc) 2001; 116: 686-691.

29. Molina M, RABAdÁn JA. Manual básico de nefrología para atención primaria. Murcia: Ed. La Gráfica, 2000.

30. Heart Outcomes Prevention Evaluation (HOPE) Study Investigators. Effects of ramipril on cardiovascular and microvascular outcomes in people with diabetes mellitus: results of the HOPE study and MICROHOPE substudy. Lancet 2000; 355: 253-259.

31. Hansson L, Zanchetti A, CARruthers SG, DAHLOF B, ELMFELDT D, Julius S et al. Effects of intensive blood-pressure lowering and lowdose aspirin in patients with hypertension: principal results of the hypertension optimal treatment (HOT) randomised trial. HOT Study Group. Lancet 1998; 351: 1755-1762.

32. Hernández H, Brito O, Domínguez A, Luengas M, Almada J, Hernández H. Prevalencia de factores de riesgo coronario en pacientes hipertensos. Rev Mex Cardiol 1999; 10: 112117.

33. Assmann G, Schulte H. The prospective cardiovascular Munster (PROCAM study): prevalence of hyperlipidemia in persons with hypertension and or diabetes mellitus and the relationship to coronary heart disease. Am Heart J 1988;116:1713-1724.

34. Plaza I, Villar F, Mata P, Pérez F, Maiquez A, CASASnOVAS JA et al. Control de la colesterolemia en España. Un instrumento para la prevención cardiovascular. Rev Esp Cardiol 2000; 53: 815-837.

35. Aranda P, Banegas JR, Barajas R, Luque M, RodICIO JL. Diagnosis and therapeutic management of hypercholesterolemic spanish hypertensives. J Hypertens 1999; 17 (Supl 3): 192.

36. KAYSEN GA. Hyperlipidemia of nephrotic syndrome. Kidney Int 1991; 39 (Suppl 31): S8S15.

37. Newmark SR, Anderson CF, Donadio JV, ElleFSON RD. Lipoprotein profiles in adult nephrotic. Mayo Clin Proc 1975; 50: 359-366.

38. KLAHR S, SchreINER G ICHIKAWA I. The progression of renal disease. N Engl J Med 1988; 318 : 1657-1666.
39. KuUsisto J, MikKÄNEn L, PyöRÄLÄ K, LAAKSO M. Hyperinsulinemic microalbuminuria. A new rosk indicator for coronary heart disease. Circulation 1995; 91: 831-837.

40. Doerks GF, Boven AJ, Hillege HL, Jansen WM, Kors JA, JONG PE et al. Microaluminuria was associated with ischemic electrocardiographic abnormalities in a large non-diabetic population. The PREVEND (Prevention of Renal and Vascular Endstage Disease). Eur Heart J 2000; 21: 1922-1927.

41. Jensen JS, Feldt B, Borch K, Clausen P, AppleYARD M, JENSEN G. Microalbuminuria and its relation to cardiovascular disease and risk factors. A population-based study of 1.254 hypertensive individuals. J Hum Hypertens 1997; 11: 727-732.

42. Pontremoli R, Sofía A, Ravera M, Nicolella C, ViazZi F, TirotTA A et al. Prevalence and clinical correlates of microalbuminuria in essential hypertension. The MAGIC study. Hypertension 1997; 30: 1135-1143.

43. Staessen JA, Fagard R, Thiss L. The systolic hypertension. Europe trial investigators. Morbidity and motality in the placebo controlled European trial of isolated systolic hypertension in the elderly. Lancet 1997; 360: 755-764.

44. ApPlEgate WB, Sowers JR. Elevated systolic blood presure: increased cardiovascular risk and rationale for treatment. Am J Med 1996 (Supl. 3A): 3S-9S.

45. HAFFNER SM: Obesity and the metabolic sindrome: The San Antonio heart study. 44: Br J Nutr 2000; 83 (Supl 1): 67-70.

46. KANNEL WB. Fifty years of Framingham Study contributions to understanding hypertension. J Hum Hypertens 2000; 14: 83-90.

47. Chen W, SRinivassan SR, ElKasabany A, BerenSON GS. The association of cardiovascular risk factor clustering related to insulin resistance syndrome between young parents and their offspring: The Bogalusa Heart Study. Atherosclerosis 1999; 145: 197-205.

48. Scholze J, Sharma AM. Treatment of hypertension in obesity. Herz 2001; 26: 209-221.

49. Mulyadi L, Stevens C, Munro S, Lingard J, BERMINGHAM M. Body fat distribution and total body fat as risk factors for microalbuminuria in the obese. Ann Nutr Metab 2001; 45: 67-71.

50. Agrawal B, Wolf K, Berger A, Luft FC. Effect of antihypertensive drugs on microalbuminuria in non-diabetic patients with essential hypertension. J Hum Hypertens 1996; 10: 551-555. 
51. Rovira E, Julve R, Pascual JM, Miralles A, REDON J. Estudio de los factores relacionados con los cambios en la microalbuminuria durante el tratamiento antihipertensivo. Med Clin (Barc) 2000; 114: 721-725.
52.2003 European society of hypertensioneuropean society of cardiology guidelines for the management of arterial hypertension. J Hypertension 2003; 21: 1011-1053. 